\title{
Zaltoprofen loaded solid lipid nanoparticles for topical delivery: formulation design, In Vitro and Ex Vivo evaluation
}

\begin{abstract}
The objective of present study is development and evaluation of solid lipid nanoparticles (SLNs) of Zaltoprofen (ZLT) for topical delivery to formulate topical formulation with prolonged effects. ZLT loaded SLNs (ZLT-SLN) were formulated using Taguchi design of Experiment and the effect of lipid ratio, surfactant ratio, stirring time and concentration of surfactant was studied on entrapment efficiency. The optimized formulation was spherical in shape with mean particle size distribution of $208 \mathrm{~nm}$ and zeta potential of $-25 \mathrm{mV}$. They were characterized for structural changes using Differential Scanning Colorimetry and Fourier Transform Infrared Spectroscopy. Optimized nanoparticles were further incorporated in carbopol hydrogel and characterized for $\mathrm{pH}$, homogeneity, viscosity and spreadability. In vitro and Ex vivo studies were carried out for ZLT-SLN loaded carbopol gel and compared against ZLT-SLN suspension. ZLT-SLN suspension followed Higuchi cube root model whereas ZLT-SLN loaded gel followed Korsemeyer Peppas model in in vitro as well as ex vivo studies which may be due to increase in viscosity. SLN loaded gel showed sustained release as compared to plain drug loaded hydrogel confirming that SLN are efficient to encapsulate ZLT and effective for sustained release of drug.
\end{abstract}

Keywords: zaltoprofen, solid lipid nanoparticles, topical delivery, solvent injection method
Volume 4 Issue 2 - 2017

\author{
Vaishali Londhe, Swarali Save \\ SVKM's NMIMS SPPSPTM, India
}

Correspondence: Vaishali Londhe, Pharmaceutics, SVKM's NMIMS SPPSPTM,Vile Pale (W), Mumbai 400056, India, Fax +91-22-26185422, Tel +919167745846,

Email vaishali.londhe@nmims.edu

Received: June 15,2017 | Published: November 16,2017
Abbreviations: ZLT, zaltoprofen; BCS, bio-pharmaceutics classification system; DDS, drug delivery system; FTIR, fourier transformed infrared; Gm, gram; HCL, hydrochloric acid; Hrs, hour; IP, indian pharmacopeia; JP, japanese pharmacopeia; LOD, loss on drying; SLNs, solid lipid nanoparticles; GMS: glycerol monostearate; $\mathrm{DRG}$, dorsal root ganglion

\section{Introduction}

The number of new pharmaceutical compounds with poor aqueous solubility has grown steadily over the last two decades due to the progress in discovery technologies, e.g. high throughput and combinatorial screening tools, during lead development and optimization phase. Most of these compounds are mainly categorized as bio pharmaceutics classification system (BCS) class II for which their oral absorption is limited by their extremely poor solubility in the gastrointestinal tract. ${ }^{1}$ Many potential compounds frequently drop out of pharmaceutical development process because of their insufficient bioavailability. Therefore, the improvement of solubility and dissolution of poorly water-soluble drugs has become vital for formulation researchers. ${ }^{2}$ Delivering the drug accurately and safely to its target site at precise time in order to have a controlled release and achieve maximum therapeutic effect remains a benchmark in the design and development of newer drug delivery systems. Nanoparticles aid to provide targeted delivery of many drugs, to sustain the effect of drug in target tissue, to improve oral bioavailability and to increase the stability of therapeutic agents against degradation. ${ }^{3}$

SLNs are colloidal carrier system composed of a high melting point lipid as a solid core coated by aqueous surfactant and the drugs used are of BCS Class II and IV. ${ }^{4}$ Solid lipid nanoparticles (SLN) represent a promising approach as a drug delivery system for topical application. ${ }^{5,6}$ Similar studies have been performed on number of drugs like Meloxicam, ${ }^{7}$ soy isoflavones, ${ }^{8}$ flurbiprofen ${ }^{9}$ and Ibuprofen, ${ }^{10}$ was formulated with the aim of better penetration through stratum cornea and efficient deposition of drug in dermal matrix. Further they were evaluated for in vitro drug release characteristics, rheological behaviour, pharmacokinetic and pharmacodynamics studies and showed excellent physical stability, high entrapment efficiency and controlled drug release can be produced representing a promising carrier for topical delivery of drugs. ${ }^{11}$

Zaltoprofen (2-(10-oxo-10, 11, dihydrodibenzo [b, f] thiepin-2yl) propionic acid belongs to the class of NSAIDs, a low molecular weight drug that acts by inhibiting the bradykinin-induced responses by blocking bradykinin interaction with the bradykinin $\beta_{2}$ receptor on dorsal root ganglion (DRG) neurons. ${ }^{11}$ Recent study on Zaltoprofen opened up its potential to be delivered as nanosuspension, ${ }^{12}$ Nanoemulsion gel ${ }^{13}$ and as drug loaded biopolymeric nanoparticles. ${ }^{14}$ All these results suggest that zaltoprofen possesses novel antiinflammatory mechanism, which inhibits $\beta_{2}$-type BK receptor function in nerve endings and shows potential to be delivered using novel drug delivery systems.

\section{Materials and methods}

\section{Materials}

Zaltoprofen API was received as a gift sample from IPCA Laboratories, Mumbai. Glycerol Monostearate and Tween 80 were purchased from Loba Chemie, Poloxamer 188 was obtained from BASF, Methanol and Acetonitrile (HPLC grade) were purchased from SRL Fine chemicals, Carbopol 940, Triethanolamine, Methyl paraben, Propyl paraben were purchased from SD Chemicals. Millipore water was used throughout the study. 


\section{Screening of lipids and solvents}

The aim of this study was to develop a solid lipid nanoparticle for topical formulation. Considering solubility of drug in lipids as one of the most important criteria, lipid and solvents were selected cautiously. The solubility of drug in lipid phase can affect the ability of the nanosuspension to maintain the drug in a solubilized form which is important in maintaining stability. The solubility of ZLT in various lipids was determined by adding an excess amount of drug in $100 \mathrm{mg}$ of selected lipid (Stearic acid, Glycerol monostearate, Compritol 888 ATO, Precirol 888 ATO and Lipoid S-75), in $10 \mathrm{~mL}$ capacity beaker using a magnetic stirrer. Similarly different volatile organic solvents (Acetone, Dichloromethane, Ethanol and Methanol) exhibiting good solubility for zaltoprofen were screened for its ability to solubilize the lipid.

\section{Preparation of solid lipid nanoparticles}

Based on the solubility studies of drug, Glycerol monostearate ${ }^{15}$ was selected as the lipid phase and Methanol as solvent. Tween 80 and poloxamer 188 were selected as surfactant and stabilizer respectively. Millipore water was used as an aqueous phase for optimization. Several batches of SLNs were prepared using different combinations of lipid and surfactant ratio. Modified Solvent Injection Method ${ }^{9,16}$ was used to formulate ZLT loaded solid lipid nanoparticles. Glycerol monostearate (GMS) and drug Zaltoprofen were dissolved in methanol and warmed to $70 \pm 2^{\circ} \mathrm{C}$. Tween 80 and Poloxamer 188 were dissolved in distilled water to prepare aqueous phase. The organic phase of alcoholic solution containing lipid mixture was added drop wise to pre-warmed aqueous solution $\left(70 \pm 2^{\circ} \mathrm{C}\right)$ with the help of a hypodermic needle on a magnetic stirrer for varying time periods to obtain nanoparticles with optimum size.

\section{Optimization of solid lipid nanoparticles}

In this experiment, Taguchi design (34) was adopted to optimize ZLT-SLN formulation process in order to derive optimal conditions. The trial studies were based on three levels (high, medium, low). Drug: Lipid ratio, Lipid: Surfactant ratio, Concentration of stabilizer and stirring time were considered as independent factors. Entrapment efficiency was considered as dependent factor in the proposed study. All statistical studies were performed using Design-Expert 9.0 software, manufactured by Stat-Ease, Inc. Analysis of variance was carried out to determine the significance of the fitted equation. The formulation was chosen with the criteria of high entrapment efficiency and absence of sedimentation. The process variables, their coded experimental values, are listed in Table 1 .

Table I Design for optimization of solid lipid nanoparticles using Taguchi design of experiment

\begin{tabular}{lllll}
\hline Batch no & Factor I & Factor 2 & Factor 3 & Factor $\mathbf{4}$ \\
\hline & Drug: lipid ratio & Lipid: surfactant ratio & Conc of stabilizer (\%) & Stirring time (Hours) \\
\hline ZLT001 & $01: 05$ & $01: 00.5$ & $0.10 \%$ & 4 \\
ZLT002 & $01: 05$ & $01: 02$ & $0.50 \%$ & 8 \\
ZLT003 & $01: 07$ & $01: 01$ & $0.10 \%$ & 8 \\
ZLT004 & $01: 06$ & $01: 00.5$ & $0.30 \%$ & 8 \\
ZLT005 & $01: 07$ & $01: 00.5$ & $0.50 \%$ & 6 \\
ZLT006 & $01: 05$ & $01: 01$ & $0.30 \%$ & 6 \\
ZLT007 & $01: 06$ & $01: 01$ & $0.50 \%$ & 4 \\
ZLT008 & $01: 06$ & $01: 02$ & $0.10 \%$ & 6 \\
ZLT009 & $01: 07$ & $01: 02$ & $0.30 \%$ & 4 \\
\hline
\end{tabular}

\section{Characterization of solid lipid nanoparticles}

Entrapment efficiency: $2 \mathrm{ml}$ of solid lipid nanoparticles were centrifuged at $23500 \mathrm{rpm}$ for 90 minutes. The free drug content in supernatant after centrifugation was measured by Ultraviolet spectroscopy. ${ }^{17}$ The calibration curve of absorbance against concentration of Zaltoprofen was plotted. Equation obtained from the graph is shown below:

$$
y=0.0126 x-0.0835, r^{2}=0.9996
$$

where;

$\mathrm{y}=$ Absorbance,

$\mathrm{x}=$ Zaltoprofen concentration

From the above equation, entrapment efficiency was calculated based on given formula:

\section{$\%$ Entrapment Efficiencey $=($ Amount of drug added - Amount of drug free in supernatant $\times 100) /($ Amount of drug added $)$}

Measurement of particle size: Photon co-relation spectroscopy using Zetasizer (Malvern Instruments, UK) determined globule size of the nanoemulsion by the principle of fluctuation in light scattering due to Brownian motion of the particles. ${ }^{18}$

Measurement of zeta potential: Zetasizer (Malvern Instruments) was used to measure Zeta Potential. ${ }^{18}$ Samples were prepared for analyzing zeta potential by diluting $1 \mathrm{ml}$ of the SLN dispersion with $10 \mathrm{ml}$ Millipore water.
Transmission electron microscopy: The morphology of particles was examined by TEM (Hitachi 2000 Japan). Combination of bright field imaging at increasing magnification and of diffraction modes was used to reveal the form and size of the nanosuspension. ${ }^{18}$

Differential scanning calorimetry: Scan of about $2 \mathrm{mg}$, accurately weighed zaltoprofen and ZLT-SLN formulation using a differential scanning calorimeter was recorded. Sealed and perforated aluminium pans were used in experiments for all the samples. Sample was run at the scanning rate of $10^{\circ} \mathrm{C} /$ minute from $30-150^{\circ} \mathrm{C}$. 
Incorporation of solid lipid nanoparticles into gel: $1 \% \mathrm{w} / \mathrm{w}$ Carbopol 940 was dispersed in drug loaded solid lipid nanosuspension. Carbopol 940 was incorporated in nanosuspension and soaked overnight. The formulation was stirred with alkalinizing agent triethanolamine till $\mathrm{pH}$ 6 and the gel of desired viscosity is obtained..$^{19,20}$

Characterization of gel: ZLT-SLN loaded gel was characterized for drug content, $\mathrm{pH}$, Homogeneity, Viscosity and Spreadability. Every test was performed in triplicate. ${ }^{21}$

Drug content: Gel equivalent to $100 \mathrm{mg}$ of ZLT was taken and dissolved in $100 \mathrm{~mL}$ of methanol in a volumetric flask. Gel solution was subjected to shaking for $2 \mathrm{~h}$ on mechanical shaker to obtain complete solubility of drug. The solution was estimated spectrophotometrically at $331 \mathrm{~nm}$ using methanol as solvent.

pH of gel:1 $\mathrm{gm}$ of gel was taken and dissolved in $100 \mathrm{ml}$ of purified water and stored for two hour. The three samples were checked for $\mathrm{pH}$ using $\mathrm{pH}$ meter (Lab India).

Homogeneity: All developed gels were tested for homogeneity by visual inspection after the gels have been set in the container.

Viscosity: Brookfield digital viscometer was used to measure the viscosity of prepared gel formulations. The spindle no. 6 was rotated at $10 \mathrm{rpm}$. The reading, near to $100 \%$ torque was noted. Samples were measured at $30 \pm 1^{\circ} \mathrm{C}$.

Spreadability study of topical gel: Spreadability was determined using apparatus consisting of wooden block and 11 pulleys at bottom. 'Slip' and 'Drag' principle were used to measure the spreadability of gel. About $2 \mathrm{gm}$ of gel was placed on ground slide. Another glass slide was placed on ground slide and entrapped air between the slides was removed. $1 \mathrm{Kg}$ of weight was put on the slide for 5 minutes. Excess of gel was cleaned from the corner of slide $80 \mathrm{gm}$ weight was placed on the pulley and time required by the top slide to move a distance of $7.5 \mathrm{~cm}$ was reported. A short interval indicates better spreadability. ${ }^{22}$ Spreadability was then calculated using the following formula:

$S=M \times L / T$

Where,

S: Spreadability,

M: Weight in the pan (tied to the upper slide),

L: Is the length moved by the glass slide and

T: The time taken to separate the slide completely from each other

In vitro drug release study: In vitro release studies were performed using modified Franz diffusion cells. Dialysis membrane having pore size $2.4 \mathrm{~nm}$ molecular weight cut off 1200-14000 (HIMEDIA) was used. Membrane was soaked in phosphate buffer $\mathrm{pH} 6.8$ overnight. Zaltoprofen formulation was filled in donor compartment and the recipient compartment was filled with phosphate buffer $\mathrm{pH}$ 6.8. The content of the cell was stirred with the help of magnetic stirrer at $32^{\circ} \mathrm{C}$. At fixed time interval predetermined aliquots of sample was withdrawn from receiver compartment through side tube. Fresh phosphate buffer of $\mathrm{pH} 6.8$ was used to replace the withdrawn medium to maintain sink condition. Samples were analyzed by UV spectrophotometer. ${ }^{23}$

Ex vivo drug release study: Ex vivo release studies were performed using modified Franz diffusion cells. Porcine ear skin was used. Skin was soaked in phosphate buffer pH 6.8 overnight. Zaltoprofen formulation was filled in donor compartment was filled in donor compartment and the recipient compartment was filled with phosphate buffer $\mathrm{pH}$ 6.8. The content of the cell was stirred with the help of magnetic stirrer at $32^{\circ} \mathrm{C}$. At fixed time interval predetermined aliquots of sample was withdrawn from receiver compartment through side tube. Fresh phosphate buffer of $\mathrm{pH} 6.8$ was used to replace the withdrawn medium to maintain sink condition. Samples were analyzed by UV spectrophotometer. ${ }^{23}$

Drug diffusion kinetics study: The in vitro and ex vivo release pattern was evaluated to study flux .Mass transport of molecules in a solution or molecular transport across a barrier is normally measured by flux. The flux of a solute is simply defined as the mass or number of molecules moving through a given cross-sectional area during a given period of time:

$$
J=m / A t
$$

Where;

\section{$\mathrm{J}$ : Is the flux of a mass of compound}

$\mathrm{m}$ : Moving through a cross-sectional area

\section{At: During time t.}

The unit for a flux value could thus be mol $\mathrm{cm}^{-2} \min ^{-1}$, or alternatively mol cm- ${ }^{2} \mathrm{~min}^{-1}$, or alternatively $\mathrm{mg} \mathrm{cm}-{ }^{2} \mathrm{~h}^{-}{ }^{1} .{ }^{21}$

The in vitro and ex vivo release pattern was evaluated to check the goodness of fit to the zero order kinetics, first order kinetics, Higuchi's square root of time equation, Hixson Crowell cube root equation, Korsmeyer Peppas power law equation.

Stability studies: The optimized SLN loaded gel formulation was stored at $40^{\circ} \mathrm{C} / 75 \% \mathrm{RH}, 4^{\circ} \mathrm{C}$ and room temperature for a period of three months and in vitro release was determined to evaluate its stability.

\section{Results and discussion}

\section{Screening of lipids and solvent}

To assess solubility of Zaltoprofen, various lipids were screened. Steric acid, Glycerol Monostearate, Compritol 888 ATO, Precirol 888 ATO and Lipoid S-75 were chosen from preliminary screening of lipids. Zaltoprofen showed excellent solubility in Glycerol monostearate and Lipoid S-75 having a melting point of $60^{\circ} \mathrm{C}$ and $40^{\circ} \mathrm{C}$ respectively. Zaltoprofen showed good solubility in Acetone, Ethanol, Methanol and Dichloromethane. Depending on solubility of the lipids in solvent, Methanol was selected as a solvent. Solid lipid nanoparticles were formulated using Solvent Injection Emulsification and Evaporation technique using Glycerol monostearate and Lipoid S-75 as lipid. Formulation with Lipoid S-75 showed larger particle size (2 microns) than that with Glycerol monostearate (400nm). Latter showed an acceptable particle size. Therefore Glycerol monostearate was selected as lipid, Tween 80 as surfactant, poloxamer 188 as a stabilizer, Methanol as solvent and water as continuous phase.

\section{Analysis of optimization design}

In the present study different formulation batches were prepared on the basis of independent factors including concentration of lipid, surfactant, stabilizer and stirring time and were analyzed for maximum entrapment efficiency. Taguchi's Design of Experiment was adopted 
for optimization studies which listed a run of nine designs. Shapiro Wilk test showed $p$ value more than 0.1 with no terms selected, which meant that there are no statistically significant differences on studied effect. Hence it may be concluded that no factors were significantly affecting entrapment efficiency of Solid Lipid Nanoparticles for Zaltoprofen. The formulation batches exhibiting high entrapment efficiency were selected for further screening. The three formulations were studied for average particle size (Table 2). From the above results Batch no. ZLT007 with an average particle size of 208nm (Table 3).

Table 2 Results for entrapment efficiency to optimize the solid lipid nanoparticles formulation

\begin{tabular}{lll}
\hline Batch no & \% Entrapment efficiency $(\% w / w)$ & Sedimentation \\
\hline ZLT00I & 65 & Observed \\
ZLT002 & 48.1 & Observed \\
ZLT003 & 64.35 & Not Observed \\
ZLT004 & 35.62 & Observed \\
ZLT005 & 55.43 & Not Observed \\
ZLT006 & 65.5 & Not Observed \\
ZLT007 & 70.6 & Not Observed \\
ZLT008 & 33.21 & Not Observed \\
ZLT009 & 46.43 & Observed \\
\hline
\end{tabular}

\section{Characterization of optimized batch}

Batch no. ZLT007 with an average entrapment of $70.6 \%$ and average particle size of $208 \mathrm{~nm}$ and polydispersity index 0.456 was optimized formulation. Zeta potential of optimized batch was found to be $-25 \mathrm{mV}$ indicating stability of the formulation.

\section{Transmission electron microscopy}

TEM was conducted to investigate the morphology of Zaltoprofen loaded SLNs. It was evident from TEM images that nanoparticles were almost spherical with smooth morphology, well dispersed and separated on the surface (Figure 1).

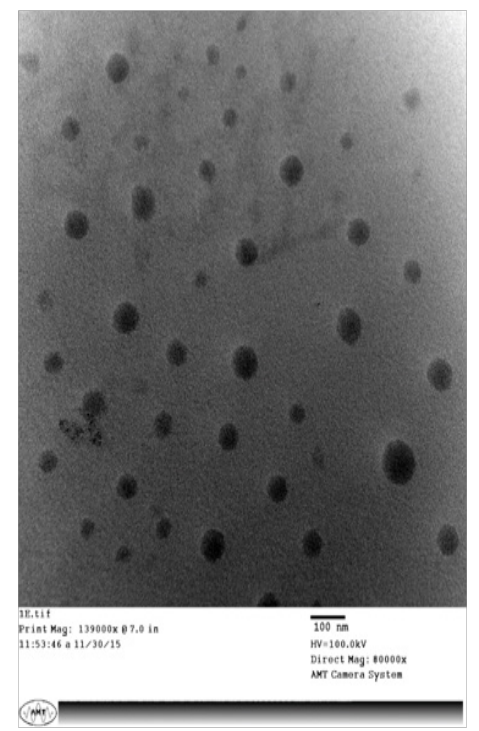

Figure I Images obtained using transmission electron microscopy of zaltoprofen loaded solid lipid nanoparticles.
Table 3 Results for particle size analysis to optimize the solid lipid nanoparticles formulation

\begin{tabular}{lll}
\hline Batch & Particle size & Polydispersity index \\
\hline ZLT003 & $277.7 \mathrm{~nm}$ & 0.608 \\
ZLT006 & $359.6 \mathrm{~nm}$ & 0.672 \\
ZLT007 & $208.0 \mathrm{~nm}$ & 0.456 \\
\hline
\end{tabular}

\section{Differential scanning calorimetry}

DSC is a tool to investigate the melting and recrystallization behavior of crystalline material like SLNs. Figure 2 and 3 shows the DSC thermograms of pure Zaltoprofen and Zaltoprofen loaded SLNs respectively. Plain Zaltoprofen showed a sharp endothermic peak at $136.6^{\circ} \mathrm{C}$ corresponding to its melting point, indicating its characteristic crystalline nature. As observed in Figure 3 , the thermogram of ZLTSLN (ZLT007) showed no peak near $136.6^{\circ} \mathrm{C}$ as shown by plain Zaltoprofen Figure 2 indicating the conversion of crystalline ZLT to the amorphous form which confirmed complete dissolution of the drug in the molten lipid matrix.

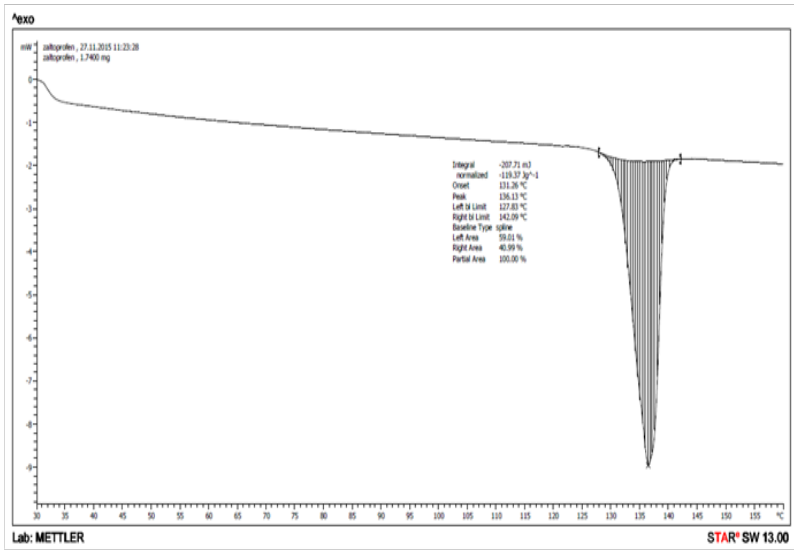

Figure 2 DSC thermogram of API zaltoprofen.

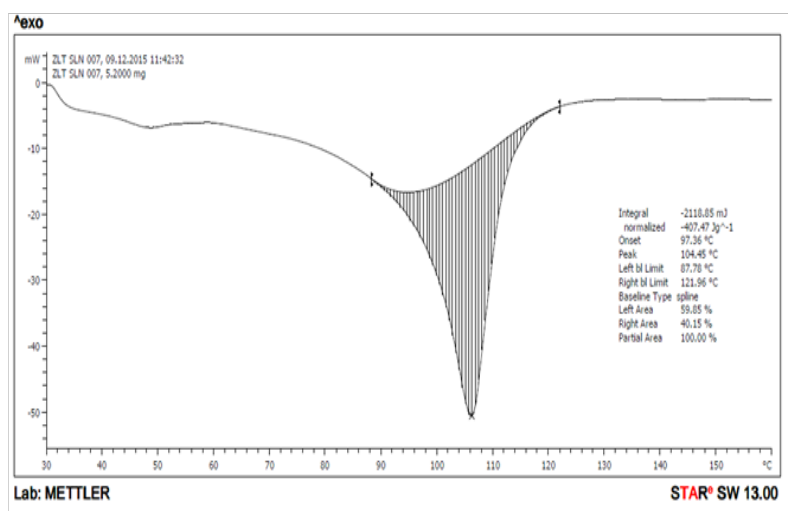

Figure 3 DSC thermogram of ZLT loaded SLN.

\section{Fourier transform infrared spectroscopy}

Figure 4 shows an overlapped FTIR spectra of Placebo solid lipid nanoparticles and ZLT loaded nanoparticles. The spectra suggest that no additional peak of Zaltoprofen was seen which indicate the entrapment of API inside the lipid matrix. Absorption peaks observed for Zaltoprofen at 2980,1703,1671,1280,799,752 $\mathrm{cm}^{-1}$ are masked in the FTIR spectra observed for ZLT loaded solid lipid nanoparticles. 


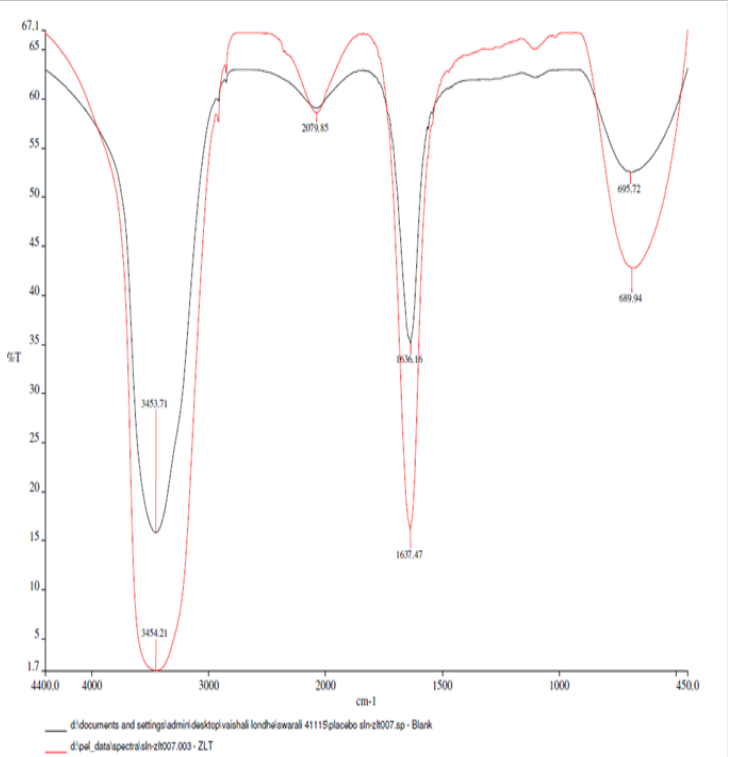

Figure 4 Comparison of FTIR spectra of placebo and zaltoprofen loaded solid lipid nanoparticles.

\section{Evaluation of SLN loaded gel}

Formulated gel appeared to be white and translucent and displayed homogeneity in texture. The $\mathrm{pH}$ of gel was observed to be $6.5 \pm 1$ which is compatible with $\mathrm{pH}$ of skin. Total drug content was noted as $90 \%$. The viscosity and spreadability of zaltoprofen solid lipid nanoparticle loaded topical gel was found to be 1898 poise and $206.8 \mathrm{gm} / \mathrm{sq} . \mathrm{cm}$ respectively

\section{In vitro diffusion studies}

The cumulative drug release from optimized formulation batch of ZLT solid lipid nanoparticles and ZLT SLN loaded gel was found to be $53.7 \%$ and $46.3 \%$ respectively. The hydrophobic drug incorporated into solid lipid showed prolonged release of drug, which improves release profile of Zaltoprofen (Figure 5).

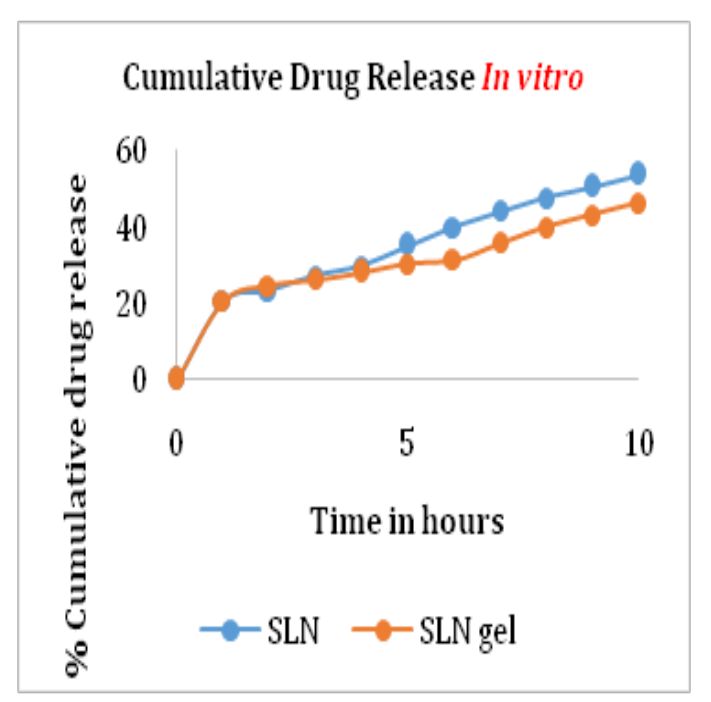

Figure 5 The cumulative amount of drug diffused from SLN and SLN gel were plotted against time in vitro.

\section{Ex vivo release study}

Ex vivo release study was performed using porcine ear skin. The cumulative drug release from optimized formulation batch of ZLT solid lipid nanoparticles and ZLT SLN loaded gel was found to be $49.6 \%$ and $36.8 \%$ respectively. The hydrophobic drug incorporated into solid lipid showed prolonged release of drug, which improves release profile of Zaltoprofen (Figure 6).

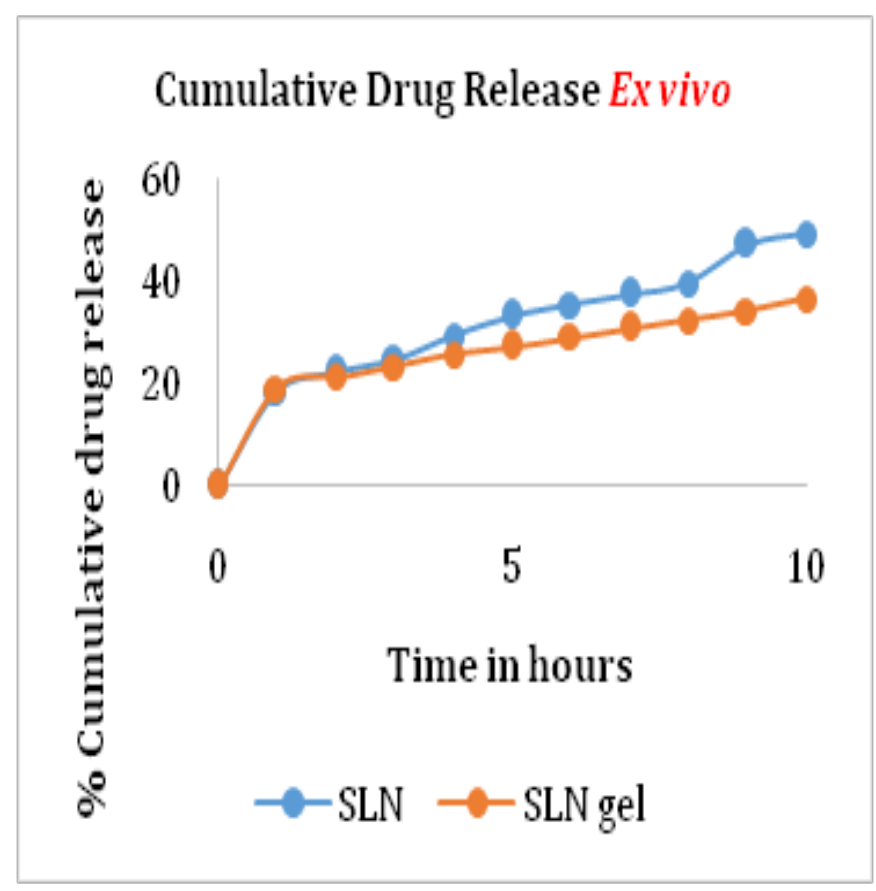

Figure 6 The cumulative amount of drug diffused from SLN and SLN gel were plotted against time ex vivo.

\section{Drug diffusion kinetic study}

Mass transport across a barrier, e.g. intestinal epithelium or other pharmaceutically relevant barriers, can be caused by diffusion or migration. Transport by simple diffusion can be measured as flux, and characterized by a permeability for the transported solute. Flux calculated by equation 4.1 at $10^{\text {th }}$ hour is as given below (Table 4): The values of $\mathrm{R}^{2}$ for different mathematical models are shown in table .The $\mathrm{R}^{2}$ values for zaltoprofen loaded solid lipid nanoparticles follows Higuchi square root model and ZLT SLN loaded carbopol gel depicts that Korsemeyer Peppas model fits best (Table 5).

Table 4 Flux at $10^{\text {th }}$ hour of diffusion study

\begin{tabular}{ll}
\hline In vitro SLN & $63.67 \mu \mathrm{g} / \mathrm{cm}^{2} /$ hour \\
In vitro SLN gel & $59.53 \mu \mathrm{g} / \mathrm{cm}^{2} /$ hour \\
Ex vivo SLN & $63.67 \mu \mathrm{g} / \mathrm{cm}^{2} /$ hour \\
Ex vivo SLN gel & $56.98 \mu \mathrm{g} / \mathrm{cm}^{2} /$ hour
\end{tabular}

\section{Stability studies}

Table 6 and Figure 7. 
Table 5 Drug diffusion kinetics for In vitro and Ex vivo drug release study

\begin{tabular}{lllll}
\hline & $\begin{array}{l}\text { Zero order } \\
\text { model }\end{array}$ & $\begin{array}{l}\text { First order } \\
\text { model }\end{array}$ & $\begin{array}{l}\text { Higuchi square root } \\
\text { model }\end{array}$ & $\begin{array}{l}\text { Hixson crowell cube } \\
\text { root model }\end{array}$ \\
\hline $\begin{array}{l}\text { In vitro ZLT SLN } \\
\text { In vitro ZLT SLN }\end{array}$ & 0.94 & 0.97 & 0.98 & 0.97 \\
mel & 0.89 & 0.93 & 0.96 & 0.92 \\
$\begin{array}{l}\text { Ex vivo ZLT SLN } \\
\begin{array}{l}\text { Ex vivo ZLT SLN } \\
\text { gel }\end{array}\end{array}$ & 0.93 & 0.96 & 0.98 & 0.95 \\
\hline
\end{tabular}

Table 6 Results for stability studies of ZLT loaded SLN gel

\begin{tabular}{llll}
\hline Formulation & RT & $\mathbf{4 0} \mathbf{0}^{\circ} \mathbf{C} \mathbf{7 5} \% \mathbf{R H}$ & $\mathbf{4}^{\circ} \mathbf{C}$ \\
\hline Physical Observation & No Change & Slight Yellow & No Change \\
$\%$ Assay & $88 \%$ & $56 \%$ & $92 \%$
\end{tabular}

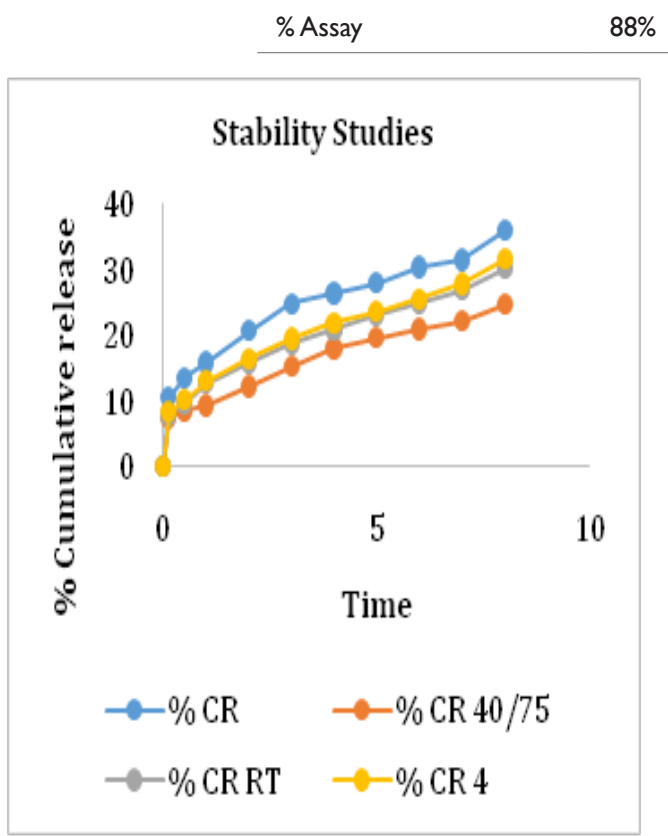

Figure 7 The cumulative amount of drug diffused from SLN gel plotted against time In vitro for stability batches.

\section{Conclusion}

The objective of the present investigation was to explore potential of Zaltoprofen loaded Solid Lipid Nanoparticles as a drug carrier system to be applied topically. Zaltoprofen, as a NSAID, was entrapped in glycerol monostearate using solvent injection, emulsification and evaporation method. The formulation was optimized by experimental design considering the concentrations of drug and lipid, surfactant, stabilizer and stirring time as independent variables. The entrapment of drug in lipid was confirmed by FTIR and DSC studies. Zaltoprofen loaded Solid lipid nanoparticles were characterized by particle size, morphology, zeta potential, and PDI and \% entrapment efficiency. The optimized design was obtained using Taguchi design. The resultant solid lipid nanoparticles were spherical with particle size of 208 $\mathrm{nm}$ and entrapment efficiency of $70.6 \%$. The -25.3 zeta potential indicated good stability of nanoparticles and PDI of 0.456 indicated aggregation free system. In vitro and Ex vivo diffusion studies showed that drug undergoes sustained diffusion from optimized formulation over a period of $10 \mathrm{~h}$. Zaltoprofen loaded solid lipid nanoparticles loaded in topical gel formulation is an alternative to tablet by virtue of its sustained and extended release and its ability to decrease side effects caused due to oral administration of Zaltoprofen. Also topical application of Zaltoprofen yields better patient compliance.

\section{Acknowledgements}

A grateful thanks to the management of Shobhaben Pratapbhai Patel School of Pharmacy \& Technology Management, NMIMS, Mumbai for encouraging and providing research facility. Also grateful to Piramal Healthcare Laboratories, Mumbai, SAIF Punjab University, Bhanuben Nanavati College of Pharmacy and IPCA Laboratories, Mumbai for their generous help in the course of this research work.

\section{Conflict of interest}

The authors of this article certify that they have no affiliations or involvement in any organization or entity with any financial or nonfinancial interest in the subject matter discussed in the manuscripts.

\section{References}

1. Papdiwal A, Pande V, Sagar K. Design and characterization of zaltoprofen nanosuspension by precipitation method. Scholars Research Library Der Pharma Chemica. 2014;6(3):161-168.

2. De Jong WH, Geertsma RE, Roszek B. National Institute for Public Health and the Environment (RIVM). Bilthoven, Netherlands; 2005.

3. Loxley A. Solid Lipid Nanoparticles for the Delivery of Pharmaceutical Actives. Drug Delivery Technology. 2009;9(8):1-5.

4. Yadav N, Khatak S, Singh S. Solid lipid nanoparticles - a review. International Journal of Applied Pharmaceutics. 2013;5(2):8-18.

5. Müller RH, Mäder K, Gohla S. Solid lipid nanoparticles (SLN) for controlled drug delivery - a review of the state of the art. Eur J Pharm Biopharm. 2000;50(1):161-177.

6. Khalil RM, El-Bary AA, Kassem MA, et al. Solid lipid nanoparticles for topical delivery of Meloxicam: development and in vitro characterization. $1^{\text {st }}$ Annual International Interdisciplinary Conference. AIIC, Azores, Portugal; 2013. p. 24-26.

7. Deshmukh K, Amin P. Formulation and evaluation of solid-lipid nanoparticle based $0.1 \%$ Soy isoflavone dermal gels. Journal of Pharmaceutical and BioSciences. 2013;1(1):7-18. 
8. Bhaskar K, Anbu J, Ravichandiran V, et al. Lipid nanoparticles for transdermal delivery of flurbiprofen: formulation, in vitro, ex vivo and in vivo studies. Lipids Health Dis. 2009;8:6.

9. Kumar PD, Subas CD, Subrata C, et al. Formulation and evaluation of solid lipid nanoparticles of a poorly water soluble model drug Ibuprofen. IRJP. 2012;3(12):132-137.

10. Dasgupta S, Ghosh SK, Ray S, et al. In vitro \& In vivo studies on lornoxicam loaded nanoemulsion gels for topical application. Curr Drug Deliv. 2014;11(1):132-138.

11. Tang HB, Inoue A, Oshita K, et al. Zaltoprofen inhibits bradykinin-induced responses by blocking the activation of second messenger signaling cascades in rat dorsal root ganglion cells. Neuropharmacology. 2005;48(7):1035-1042.

12. Aher SJ, Jagdale S. Preparation and characterization of zaltoprofen nanosuspension through quassi emulsification solvent diffusion. Indo American Journal of Pharmaceutical Research. 2014;4(12):5959-5965.

13. Chodhary P, Aparna C, Srinivas P. Formulation and Evaluation of Zaltoprofen Nanoemulsion gel. IJPT. 2014;6(2):6552-6571.

14. Shah HA, Patel RP. Statistical modelling of zaltoprofen loaded biopolymeric nanoparticles-Characterization and anti-inflammatory activity of nanoparticles loaded gel. Int J Pharm Investig. 2015;5(1):20-27.

15. Patel M. Development, Characterization and Evaluation of solid lipid nanoparticles as a potential anticancer drug delivery system. USA: The University of Toledo; 2012.95 p.
16. Trotta M, Debernardi F, Caputo O. Preparation of solid lipid nanoparticles by a solvent emulsification-diffusion technique. Int $J$ Pharm. 2003;257(1-2):153-160.

17. Shafiq S, Shakeel F, Talegaonkar S, et al. Development and bioavailability assessment of ramipril nanoemulsion formulation. Eur J Pharm Biopharm. 2007;66(2):227-243.

18. Sharma P, Nikam S, Chavan M. Solid Lipid Nanoparticles: A Lipid Based Drug Delivery. IPP. 2014;2(3):365-376.

19. Verma A, Singh S, Kaur R, et al. Formulation, optimization and evaluation of clobetasol propionate gel. Int J Pharm Sci. 2013;5(4):666-674.

20. Patel J, Patel B, Banwait H, et al. Formulation And Evaluation of Topical Aceclofenac Gel Using Different Gelling Agent. Int J Drug Dev Res. 2011;3(1):156-164.

21. Shetty C, Babubhai S, Pathan I. Development of Valdecoxib Topical Gels-Effect of Formulation Variables on the Release of Valdecoxib. Int J Pharm Pharm Sci. 2010;2(1):70-73.

22. Barhate SD, Potdar B, Nerker P. Development of Meloxicam sodium transdermal gel. IJPRD. 2000;2(5):1-4.

23. Cordero JA, Alarcon L, Escribano E, et al. A Comparative study of the transdermal penetration of a series of nonsteroidal anti-inflammatory drugs. J Pharm Sci. 1997;86(4):503-508. 